\section{Commentary: Multifaceted challenges with choosing between mitral valve repair and replacement for rheumatic heart disease}

Michael Silverman, MD, ${ }^{\mathrm{a}}$ and Muralidhar Padala, $\mathrm{PhD}^{\mathrm{a}, \mathrm{b}}$

Rheumatic fever continues to be a significant cause of valvular heart disease in countries in Asia, Africa, and Latin America. ${ }^{1-3}$ When the mitral valve is involved, it often presents with thickened tissue causing mitral stenosis, and sometimes with a mix of stenosis and regurgitation. ${ }^{4}$ Surgical correction of these lesions with valve repair or replacement is the only option, and consesus on whether one approach is superior to the other is lacking. While repair that preserves the native valve is beneficial in several ways, the heterogeneity in the disease severity, suitability of the valve pathology to repair, the complexity of valve reconstruction, longer cardiopulmonary bypass times, and the risk of recurrent rheumatic fever after surgery confound this choice. Replacement is a simpler option that avoids these issues, but the need for life-long anticoagulation with mechanical heart valves and the limited structural life of bioprosthetic heart valves complicates this choice. The choice of technique is further complicated by a variety of factors, including: the wide range of ages at which patients present for surgery, the unaffordability of prosthetic valve in some situations, noncompliance with postoperative medications due to lack of access to resources, recurrence of rheumatic fever, and the inability to provide redo operations to patients with failed mitral interventions. These

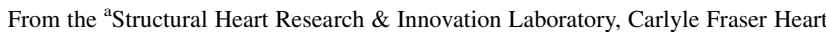
Center at Emory University Hospital Midtown; and ${ }^{\mathrm{b}}$ Division of Cardiothoracic Surgery, Emory University School of Medicine, Atlanta, Ga.

Disclosures: The authors reported no conflicts of interest.

The Journal policy requires editors and reviewers to disclose conflicts of interest and to decline handling or reviewing manuscripts for which they may have a conflict of interest. The editors and reviewers of this article have no conflicts of interest.

Received for publication Aug 10, 2020; revisions received Aug 10, 2020; accepted for publication Aug 11, 2020; available ahead of print Aug 15, 2020.

Address for reprints: Muralidhar Padala, PhD, 380B Northyards Blvd, Atlanta, GA 3031 (E-mail: spadala@emory.edu).

J Thorac Cardiovasc Surg 2022;164:70-1

$0022-5223 / \$ 36.00$

Copyright (c) 2020 by The American Association for Thoracic Surgery

https://doi.org/10.1016/j.jtcvs.2020.08.035

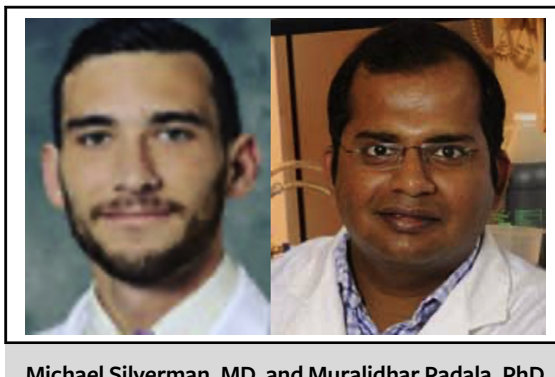

Michael Silverman, MD, and Muralidhar Padala, PhD

CENTRAL MESSAGE

Choosing between mitral valve repair and replacement in rheumatic valve disease is a decision impacted by factors well beyond the patient's anatomy or the surgeon's experience.

multifaceted challenges make the choice of approach in rheumatic mitral valve disease more challenging than the approaches considered by surgeons dealing with degenerative and functional mitral valve lesions.

Chen and colleagues ${ }^{5}$ report a retrospective, propensitymatched analysis comparing mitral valve repair with mitral valve replacement in rheumatic mitral valve disease in Taiwan. Taiwan presents a unique study environment, as it is one of the few countries that has a centralized health care system with adequate expertise in cardiac surgery, accessible health care resources, and a single-payer system. Thus, the contribution of the socioeconomic factors to the trends and outcomes is minimized in the Taiwan study population. The investigators identified 5086 patients with rheumatic heart disease who underwent mitral valve surgery, and upon propensity matching, identified 467 patients for comparison. In-hospital mortality in the repair group was $7.1 \%$ and in the replacement group was $7.3 \%$, which is not significantly different. In those surviving, patients receiving a valve repair spent less time in the intensive care unit and in the hospital than those receiving a valve replacement. Thus, the cost of mitral valve repair was significantly lower than the replacement. At a mean follow-up of 5.9 years, the all-cause mortality was 33.4\% in the repair group and $32.5 \%$ in the replacement group, which is not materially different. However, patients receiving a repair had a significantly greater rate of redo mitral valve surgery at $7.3 \%$, compared with $1.7 \%$ in the 
valve replacement group. The largest risk factor of redo mitral valve surgery is the use of percutaneous transvenous mitral commissurotomy, with other factors such as mitral stenosis-dominant anatomy, and additional surgeries on other valves governing the rates of redo surgeries. The authors further analyze the mitral valve repair group and discern that it may be suitable for rheumatic heart disease in certain conditions (eg, early stages rather than late).

The authors should be congratulated for studying their patients and reaching a conclusion that is supported by the data in the context of their health care setup. Choosing mitral valve replacement may not always be possible in other countries, and their data cannot be generalized to such situations. Despite the lucid data from this study, some limitations must be considered. First, cardiac function data are lacking from this retrospective study; thus, the relationship between mortality and functional status at presentation for surgery was not investigated. This is a significant limitation, as referral to mitral valve surgery is often delayed in this lesion, and patients may present with a highly heterogenous cardiac dysfunction. Second, the use of mitral valve repair waned over the study duration, inserting a bias into the analysis. As experience in mitral valve repair wanes and new surgeons are not trained in these techniques, the risk of poor outcomes when repair is attempted are real. Third, the follow-up duration is short compared to the timeline for structural deterioration of tissue valves, which comprised $24 \%$ of the valve replacements in this cohort. Greater reoperation rates in later years after replacement may occur, posing the same challenges seen in the repair group. The availability of transcatheter aortic valves for off-label use in the mitral position is a potential option to avoid redo surgeries, but the feasibility, efficacy, and economics of such an approach are hypothetically appealing but lacking supportive data.

Altogether, the choice of mitral valve repair or replacement may be best decided in an individualized manner, taking account of the patient's limitations, access to health care, and the surgeon's technical experience. A repair-first approach may be reasonable, but the option to convert to a replacement when the anatomy is too complicated or the dysfunction is too severe is reasonable. Advances in 3-dimensional echocardiography and computed tomography can aid such decision making and direct the surgeon along the appropriate path.

\section{References}

1. Noubiap JJ, Agbor VN, Bigna JJ, Kaze AD, Nyaga UF, Mayosi BM. Prevalence and progression of rheumatic heart disease: a global systematic review and meta-analysis of population-based echocardiographic studies. Sci Rep. 2019;9: 17022 .

2. Zuhlke L, Karthikeyan G, Engel ME, Rangarajan S, Mackie P, Cupido-Katya Mauff B, et al. Clinical outcomes in 3343 children and adults with rheumatic heart disease from 14 low- and middle-income countries: two-year follow-up of the global rheumatic heart disease registry (the REMEDY Study). Circulation. 2016;134:1456-66.

3. Remenyi B, Carapetis J, Wyber R, Taubert K, Mayosi BM, World Heart Federation. Position statement of the World Heart Federation on the prevention and control of rheumatic heart disease. Nat Rev Cardiol. 2013;10:284-92.

4. Vanderspuy JC. The clinical pathology of rheumatic mitral endocarditis. Br Heart J. 1963;25:763-70.

5. Chen S-W, Chen C-Y, Chien-Chia V, Chou A-H, Cheng Y-T, Chang S-H, et al Mitral valve repair versus replacement in patients with rheumatic heart disease. J Thorac Cardiovasc Surg. 2022;164:57-67.e11. 